\title{
MC11C4: a pilot randomized, placebo-controlled, double-blind study of venlafaxine to prevent oxaliplatin-induced neuropathy
}

\author{
Collin Zimmerman ${ }^{1}$ - Pamela J. Atherton ${ }^{2}$ - Deirdre Pachman ${ }^{2}$ • Drew Seisler ${ }^{2}$. \\ Nina Wagner-Johnston ${ }^{3}$. Shaker Dakhil ${ }^{4}$ Jacqueline M. Lafky ${ }^{1}$ - Rui Qin ${ }^{1,2}$. \\ Axel Grothey ${ }^{1} \cdot$ Charles L. Loprinzi $^{1}$
}

Received: 1 May 2015 / Accepted: 27 July 2015 / Published online: 8 August 2015

(C) Springer-Verlag Berlin Heidelberg 2015

\begin{abstract}
Purpose Previous pilot data suggested that venlafaxine could prevent acute and chronic oxaliplatin-related neuropathy. The purpose of this randomized, placebo-controlled, doubleblinded pilot study was to obtain additional data to support conducting a phase III trial to test the use of venlafaxine to prevent oxaliplatin neurotoxicity.

Methods Fifty patients, scheduled to undergo oxaliplatinbased therapy (FOLFOX) for stages II-III (67\%) or stage IV $(33 \%)$ colon cancer, were randomized to receive venlafaxine extended release $(37.5 \mathrm{mg})$ or placebo, twice daily, through their last dose of oxaliplatin and then titrated off. Neurotoxicity was evaluated via several patient- and physician-reported measures, including the European Organization for Research and Treatment of Cancer Quality of Life Questionnaire-Chemotherapy-Induced Peripheral Neuropathy 20 (EORTC QLQ-CIPN20) instrument.

Results Baseline patient characteristics were equivalent for the two arms, with a median age of 60 years. There was a trend toward benefit for the venlafaxine arm, when evaluated by the oxaliplatin-specific neuropathy scale and by acute neuropathy measures of throat discomfort and discomfort swallowing cold liquids, the latter only for the first two oxaliplatin doses. These trends were outweighed by a lack of any such trends in all other measurements including the
\end{abstract}

Charles L. Loprinzi

cloprinzi@mayo.edu

Mayo Clinic, 200 1st St SW, Rochester, MN 55905, USA

2 Alliance Statistics and Data Center, Mayo Clinic, Rochester, MN, USA

3 Washington University School of Medicine, St. Louis, MO, USA

4 Cancer Center of Kansas, Wichita, KS, USA following: (1) the CIPN20 sensory subscale ( $P=0.55$, primary endpoint), physician-completed NCI CTCAE assessment, or cumulative administered oxaliplatin doses (median 716 vs $631 \mathrm{mg}$ for placebo and venlafaxine, respectively, $P=0.34$ ).

Conclusions The present study neither supports the use of venlafaxine for preventing oxaliplatin-induced neuropathy in clinical practice nor the initiation of a phase III trial to investigate venlafaxine in this setting.

Keywords Venlafaxine $\cdot$ Chemotherapy-induced neuropathy prevention

\section{Introduction}

Oxaliplatin is widely used for the treatment of a number of malignancies and has become an integral part of chemotherapy regimens for adjuvant and metastatic colorectal cancer, particularly in combination with a fluoropyrimidine and leucovorin, as in FOLFOX [1, 2].

The dose limiting toxicity of oxaliplatin is neurotoxicity, which presents with distinct acute and chronic forms [3], thought to arise out of unique mechanisms. The acute manifestations include increased sensitivity to touching cold items or swallowing cold liquids, throat discomfort, and muscle cramping. These acute symptoms, which are manifested in $85-95 \%$ of exposed patients, peak 3 days after oxaliplatin administration; recent evidence indicates that the symptoms may not be fully reversible between cycles [4]. The chronic manifestations consist of sensory impairment of peripheral nerves with distally pronounced dysesthesias and paresthesias of the extremities, which increase in intensity with cumulative doses. These symptoms can significantly affect patient function and quality of life and may last for years after treatment completion $[1,4-6]$. 
The acute and chronic symptoms differ in clinical manifestations and are thought to arise out of distinct mechanisms. A commonly proposed mechanism for acute neurotoxicity involves the impact of oxalate, a metabolite of oxaliplatin, on $\mathrm{Na}+$ voltage-gated ion channels either via chelation of calcium and magnesium or through alteration of currents through these channels [7-9]. Chronic neurotoxicity is thought to be from a similar mechanism as occurs with other platinum agents, mediated by length-dependent axonal degeneration, mitochondrial disruption, and disruption of nerves in the dorsal root ganglion [10-12].

Effective treatments for the neurotoxicity associated with oxaliplatin are lacking [13]. In the recent past, substantial focus had been on prophylactic treatment strategies with infusional calcium and magnesium $(\mathrm{Ca} / \mathrm{Mg})$ during oxaliplatin administration [14]. This treatment approach was based on the hypothesis that infusional $\mathrm{Ca} /$ $\mathrm{Mg}$ may prevent chelation of intracellular calcium by metabolites of oxaliplatin [15]. Although $\mathrm{Ca} / \mathrm{Mg}$ had been widely used and previous clinical studies reported potential benefit with $\mathrm{Ca} / \mathrm{Mg}$ for the prevention of oxaliplatin neuropathy $[16,17]$, a recently published randomized phase III trial of 353 patients receiving FOLFOX demonstrated no statistical differences in acute or chronic neurotoxicity among those who received $\mathrm{Ca}$ / $\mathrm{Mg}$ versus placebo [18]. Thus, alternative effective means of preventing oxaliplatin-associated neuropathy are desired.

Venlafaxine, a serotonin-norepinephrine reuptake inhibitor (SNRI), is helpful for neuropathic pain caused by conditions other than chemotherapy (e.g., diabetes and zoster) [19]. A series of case studies have supported the use of venlafaxine for prevention and treatment of chemotherapy-induced peripheral neuropathy (CIPN), particularly that caused by oxaliplatin [20-22]. A small randomized placebo-controlled phase III trial (EFFOX), by Durand et al., enrolled 48 patients who had reported distressing acute neurotoxicity from oxaliplatin. Significant improvements were reported for both acute and grade 3 chronic neuropathy symptoms, in those receiving venlafaxine [23].

Based on these data, the current pilot, randomized, placebo-controlled, double-blind study was developed to further investigate the potential efficacy of venlafaxine for the prevention of oxaliplatin-induced neuropathy. It was hypothesized that results would support the findings seen in the previously reported trial [23] and provide further data to support the conduct of a larger, more definitive phase III trial.

\section{Patients and methods}

Patients considered for this clinical trial had to be adults scheduled to undergo FOLFOX chemotherapy with individual oxaliplatin doses of $85 \mathrm{mg} / \mathrm{m}^{2}$ per cycle, given in 2-week cycles. Patients had to be registered and begin this study prior to their second cycle of FOLFOX. Inclusion criteria included adequate blood counts and serum creatinine values and Eastern Cooperative Group performance status of two or better. Exclusion criteria included previous receipt of neurotoxic chemotherapy, pre-existing peripheral neuropathy of any grade, or prior use of antidepressant, anticonvulsant, or other neuropathic pain medications such as carbamazepine, phenytoin, valproic acid, gabapentin, lamotrigine, topical lidocaine patch or gel, capsaicin cream, or amifostine.

Patients were stratified according to (1) planned concurrent calcium/magnesium $(\mathrm{Ca} / \mathrm{Mg})$ administration (yes vs no), (2) colon cancer stage (II or III vs IV), and (3) study initiation cycle (prior to the first cycle vs prior to the second cycle of FOLFOX). Patients were randomly assigned, using the Pocock-Simon dynamic allocation procedure which balanced the marginal distributions of the stratification factors between the treatment groups, to venlafaxine XR $37.5 \mathrm{mg}$ twice daily vs a placebo twice daily [24]. Venlafaxine/placebo treatment was to start on the evening of the first or second chemotherapy dose and be taken through 1 week following the last dose of FOLFOX.

Patient-reported neurotoxicity and symptom evaluations were to be obtained by three patient assessments. The first is the European Organization for Research and Treatment of Cancer Quality of Life Questionnaire-ChemotherapyInduced Peripheral Neuropathy 20 instrument (EORTC QLQ-CIPN20). This 20-item CIPN-specific questionnaire includes scales assessing sensory, motor, and autonomic symptoms; it has been tested in cancer patients receiving a variety of chemotherapies and shown to have internal consistency and reliability $[25,26]$. The second is the Oxaliplatin Acute Symptom Questionnaire that has questions structured on a 0 (no problem) to 10 (major problem) numerical analog rating scale addressing "sensitivity touching cold items," "discomfort swallowing cold items," "throat discomfort," and "muscle cramps" during the previous $24 \mathrm{~h}$. While this tool has not undergone formal validation testing, it was developed using a validated method (numerical analog tool) and has been used in other publications $[17,18]$, as there is no validated patientreported outcome tool for measuring acute oxaliplatin neuropathy. The third is a supplemental symptom questionnaire that rated additional symptoms of diarrhea, abdominal cramping, constipation, bowel problems, and dysphagia, on numerical analog scales ranging from 0 to 10 .

Physician-reported evaluations were also collected. The National Cancer Institute Common Terminology Criteria for Adverse Events (NCI CTCAE) version 4 was used to classify neurotoxic symptoms and other adverse events (AEs). An Oxaliplatin Specific Neurotoxic grading scale rated the reversibility of neurotoxicity symptoms between treatment cycles 
Fig. 1 CONSORT diagram. SAE serious adverse event

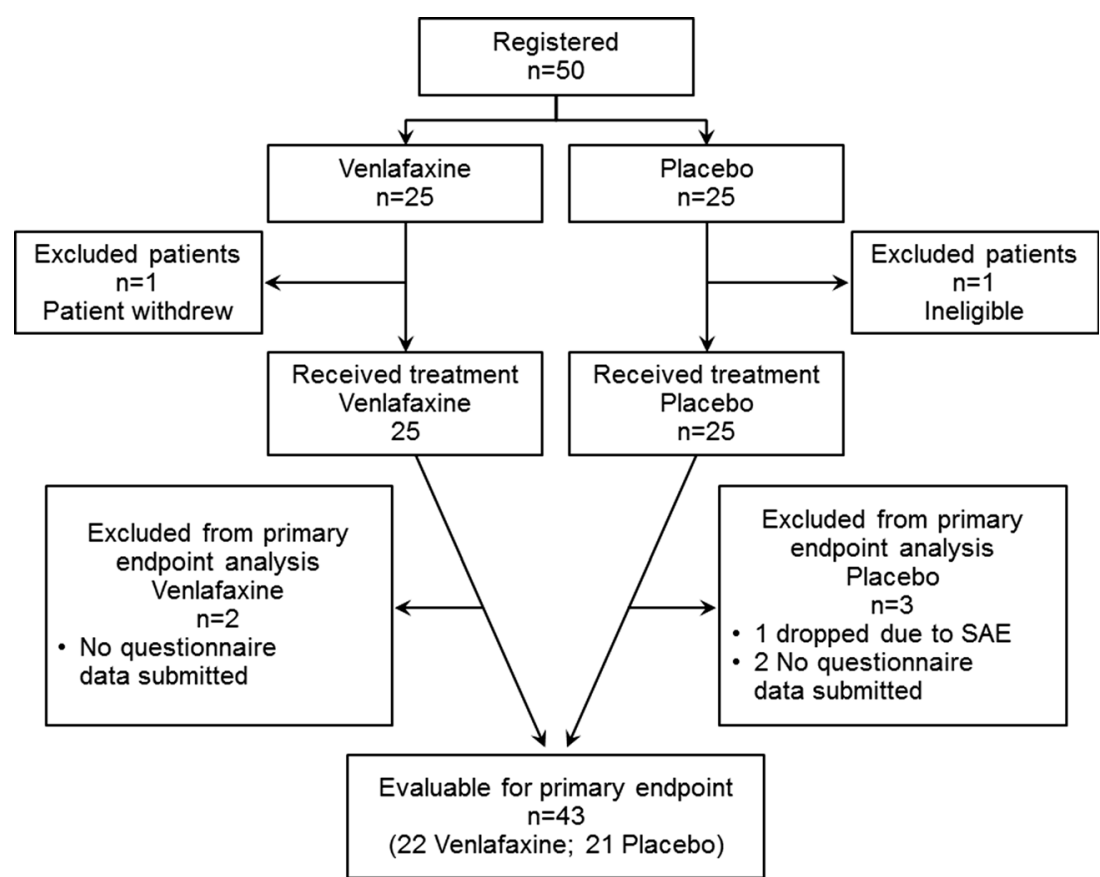

[27]. It is noted that this tool has not undergone extensive validation.

Data were also collected with regards to oxaliplatin doses administered to patients on each study arm.

History and physical exam were completed at baseline. The patient questionnaires and physician assessments were
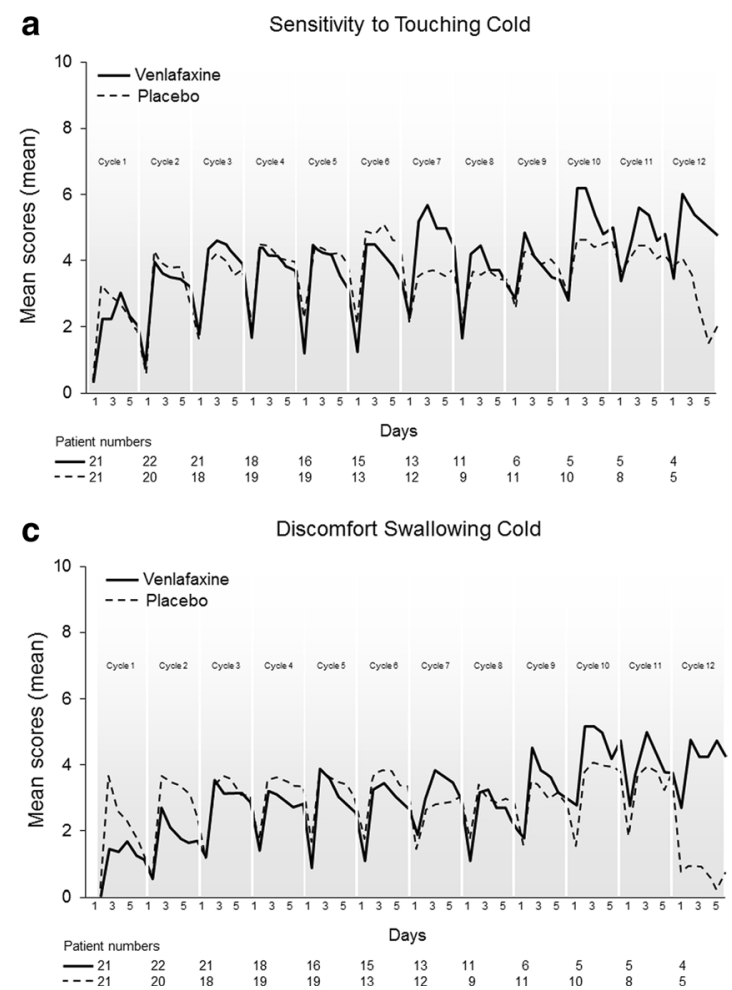

completed prior to each FOLFOX treatment. The patient questionnaires regarding chronic neuropathy were also to be completed at 1, 3, 6, and 12 months after finishing FOLFOX therapy. Additionally, the Oxaliplatin Acute Symptom Questionnaire was completed on chemotherapy treatment days and for 5 consecutive days post-chemotherapy.

b
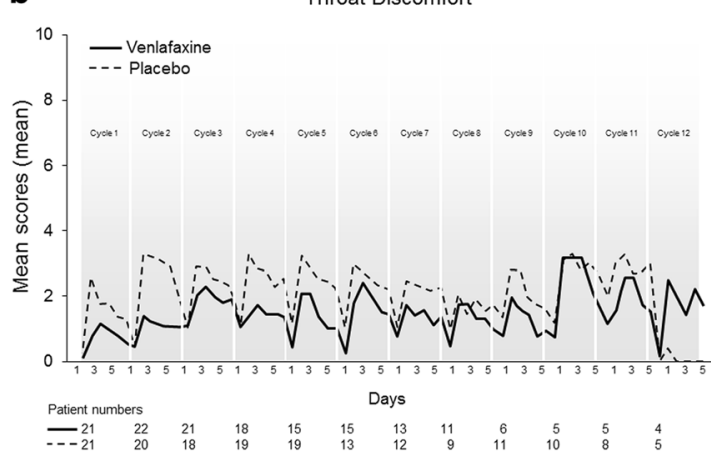

d

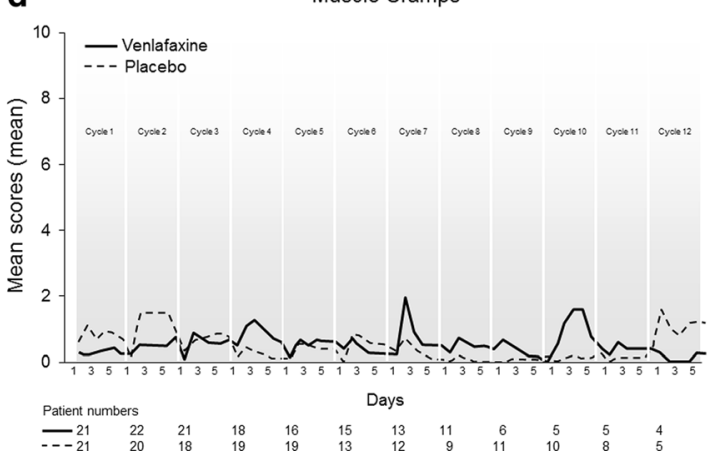

Fig. 2 Acute symptoms over time regarding sensitivity to touching cold items (a), discomfort swallowing cold liquids (b), throat discomfort (c), and muscle cramps (d). Data collected via the Oxaliplatin Acute Symptom Questionnaire; higher scores represent more symptoms 
a

CIPN20:

Sensory Neuropathy Subscale

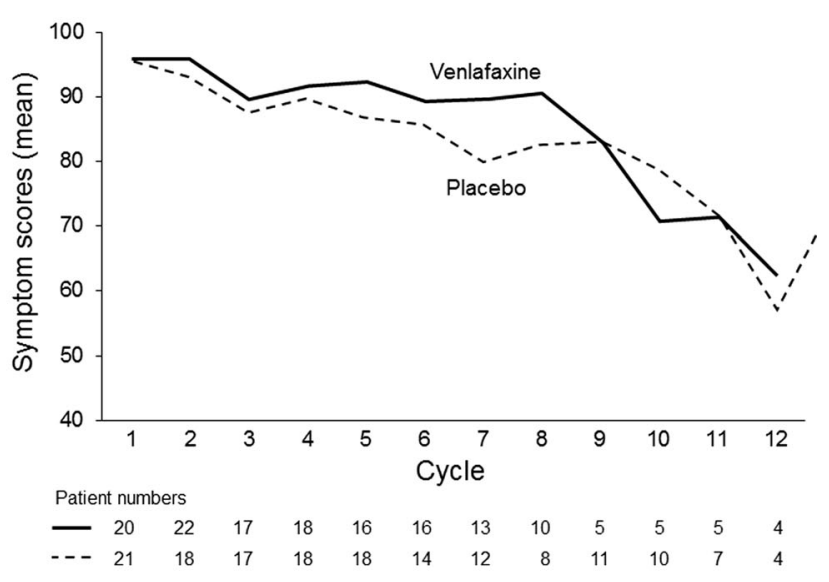

b

CIPN20 Sensory Subscale: Change from end of Treatment

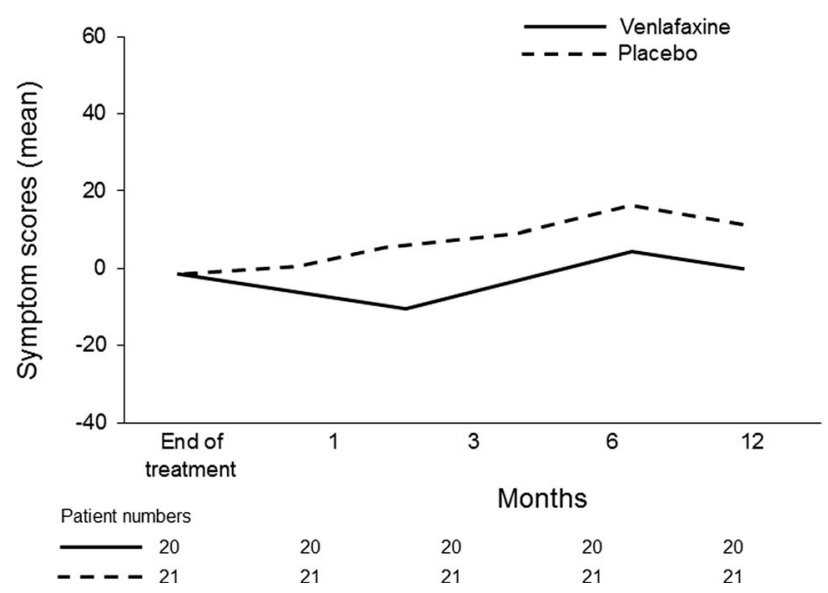

Fig. 3 Peripheral sensory neuropathy changes over time for the two study arms, as measured by the sensory scale of the EORTC QLQCIPN20 instrument, during chemotherapy (a) and following the cessation of chemotherapy (b); higher scores represent fewer symptoms

Each participant signed an IRB-approved, protocolspecific informed consent in accordance with US federal and institutional guidelines.

\section{Statistical methods}

The primary goal was to explore whether venlafaxine could prevent or ameliorate chronic neurotoxicity associated with oxaliplatin using the sensory subscale of the EORTC QLQCIPN20 measurement tool. The EORTC QLQ-CIPN20 subscales were computed according to the standard scoring algorithm and then transformed to a 0-100 scale, where high scores meant less symptom burden. The primary analysis employed longitudinal mixed effects models to assess the difference in sensory neuropathy by treatment arm over time.

The secondary and tertiary goals were to explore whether venlafaxine could ameliorate chronic motor and autonomic neurotoxicity, acute neurotoxicity, and to assess adverse events. Secondary endpoints were assessed using the motor and autonomic subscales of the EORTC QLQ-CIPN20, the CTCAE, and Oxaliplatin Specific Neurotoxic grading scale grades, and results of the individual questions of the Oxaliplatin Acute Symptom Questionnaire.

Analyses for secondary/tertiary endpoints were descriptive, and adjustments were not made for multiple comparisons due to the exploratory nature of this research. Linear growth curve models were utilized for the motor and autonomic subscales. Frequencies and percentages were calculated for discrete data and analyzed using chi-square or Fisher exact methodology. Summary statistics, including means and medians, were compiled for continuous data and analyzed using two-sided $t$ test, Wilcoxon, or Kruskal-Wallis techniques, as appropriate. Times to neurotoxic events were calculated, and KaplanMeier techniques were employed to determine differences in treatment arms.

The sample size for this pilot study was set at 50 patients ( 25 per treatment group) for logistical and financial reasons. This is similar in size to the Durand et al. trial which had 48 patients [23]. The study was designed to have the power to detect moderate differences; with 25 patients per group, we predicted that we would be able to detect effect sizes similar to what was observed in the Durand trial. In this trial, grade 3 CIPN was $0 \%$ in the venlafaxine arm versus $33.3 \%$ in the placebo arm at 3 months, and full relief of acute neurotoxicity was $31 \%$ in the venlafaxine arm versus $5 \%$ in the placebo arm in the few days after each cycle. In terms of the primary endpoint with the EORTC QLQ-CIPN20 sensory subscale, we estimated that we had a $53 \%$ power to detect an effect size of 0.5 in EORTC QLQCIPN20 at 3-month follow-up with a sample size of 50 patients.

Data collection and statistical analyses were conducted by the Alliance Statistics and Data Center.

\section{Results}

\section{Baseline characteristics}

This study accrued 50 patients from 9 individual sites between February 2012 and March 2014. Two patients, one on each arm, did not receive treatment and were excluded from the analyses. The remaining 48 patients all received the modified FOLFOX 6 regimen. Baseline patient characteristics were equivalent in the two arms. The mean age was 60.3 (placebo 58.4 , venlafaxine $62.3 ; p=0.18) ; 48 \%$ were female $(50 \%$, $46 \%$; 0.77); $94 \%$ were Caucasian (96\%, $92 \%$; 0.55); 64/ $36 \%$ of patients were receiving adjuvant/metastatic disease 
Fig. 4 Changes over time for individual questions from the EORTC QLQ-CIPN20 instrument: Do you have tingling fingers or hands? (a); Do you have tingling toes or feet? (b); Do you have numbness in your fingers or hands? (c); Do you have numbness in your toes or feet? (d); Do you have shooting or burning pains in your fingers or hands (e); Do you have shooting or burning pains in your toes or feet? (f). Higher scores represent fewer symptoms
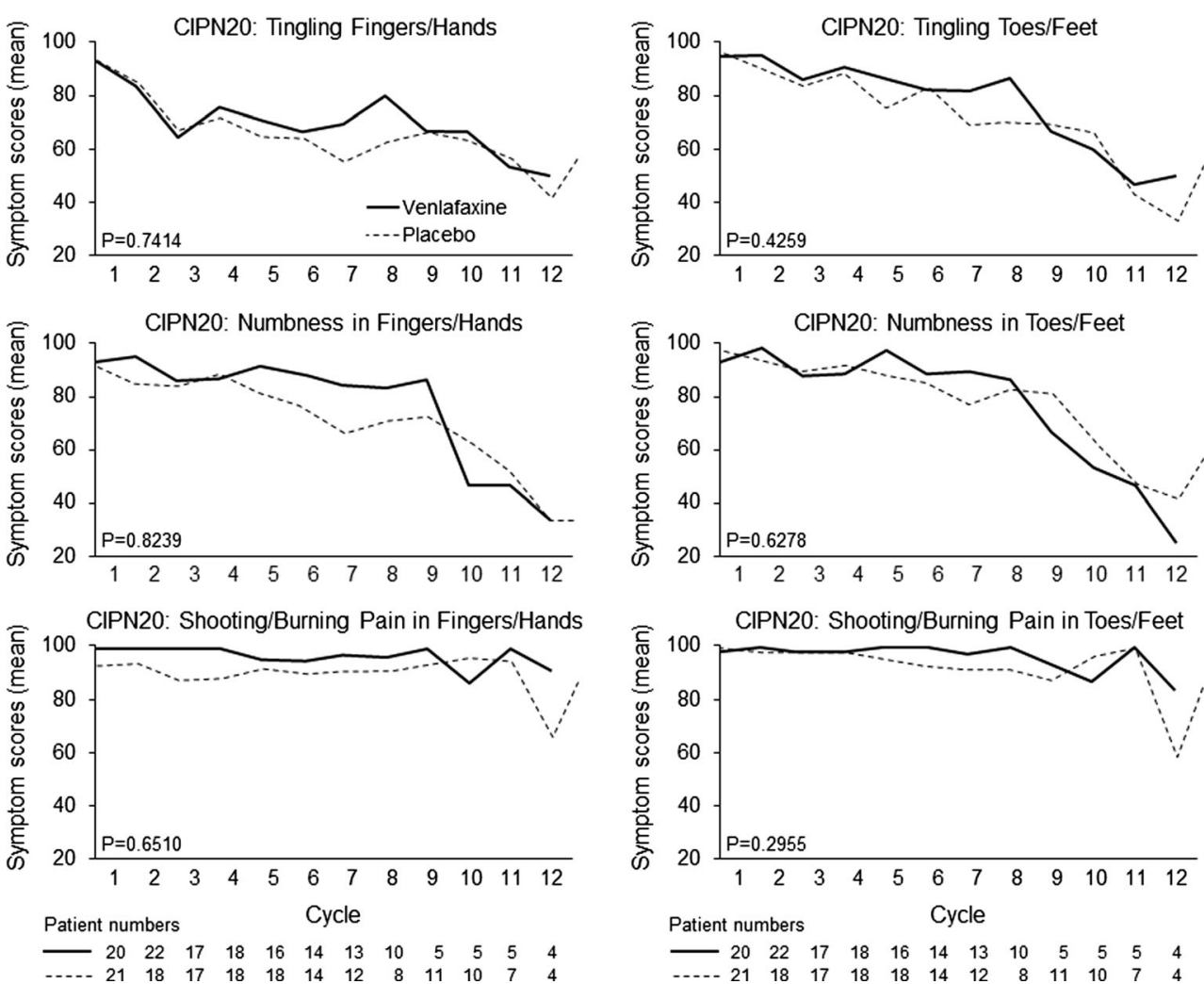

treatments $(52 / 48 \%, 74 / 26 \% ; 0.14) ; 10 \%$ were scheduled to receive concomitant intravenous calcium and magnesium to try to decrease neuropathy $(8 \%, 13 \% ; 0.64)$; and $33 \%$ had stage 4 disease $(38 \%, 29 \%$; 0.54). Patient study flow is illustrated in Fig. 1.

\section{Acute neuropathy}

Acute neuropathy data, collected via the Oxaliplatin Acute Symptom Questionnaire (Fig. 2), did not suggest that there was any substantial benefit for venlafaxine. However, with the venlafaxine arm, there appeared to be somewhat less (1) throat discomfort (Fig. 2b) and (2) discomfort swallowing cold liquids (Fig. 2c), the latter observed only for the first two oxaliplatin doses.

\section{Chronic neuropathy}

The primary endpoint of CIPN, as measured by the EORTC QLQ-CIPN20 sensory neuropathy scale and analyzed using growth curve models, demonstrates no significant differences between placebo and venlafaxine arms (Fig. 3a, $p=0.55$ ). Growth curve models using the motor and autonomic subscales of the EORTC QLQ-CIPN20 also indicated no differences between arms $(p=0.25$ and $p=0.23$, respectively; data not shown). Follow-up data, following the completion of oxaliplatin, for the sensory subscale are illustrated in Fig. $3 \mathrm{~b}$ which numerically favor the placebo arm. Data from the six individual questions of the EORTC QLQ-CIPN20 sensory scale (numbness, tingling, and shooting/burning pains in fingers/hands and in toes/feet) are illustrated in Fig. 4, again failing to illustrate any significant differences between the two study arms. Evaluation of the other 14 items in the EORTC QLQ-CIPN20 tool, likewise, did not demonstrate any suggestion of benefit for the venlafaxine arm (data not shown).

\section{Time to neurotoxic events}

When neurotoxicity was evaluated by clinician-determined measurements using CTCAE criteria, there were 11 grade $2+$ CIPN AEs in the placebo arm and 8 grade $2+$ CIPN AEs in the venlafaxine arm. Kaplan-Meier analyses indicate no significant differences between the two study arms in the time these events occurred ( $p=0.35$, Fig. 5a). Similarly, the time to grade $2+$ oxaliplatin-specific neuropathy, as measured by the Oxaliplatin Specific Neurotoxic grading scale, was not significantly different between the two treatment arms $(p=0.35$, Fig. 5b).

\section{Oxaliplatin dose delivery}

Since neurotoxicity can limit oxaliplatin dosing or influence early discontinuation, cumulative oxaliplatin dosage and discontinuation were evaluated. Figure 6 illustrates the percentage of patients continuing on oxaliplatin (Fig. 6a), percentage receiving full dose (Fig. 6b), and the mean oxaliplatin doses 
a

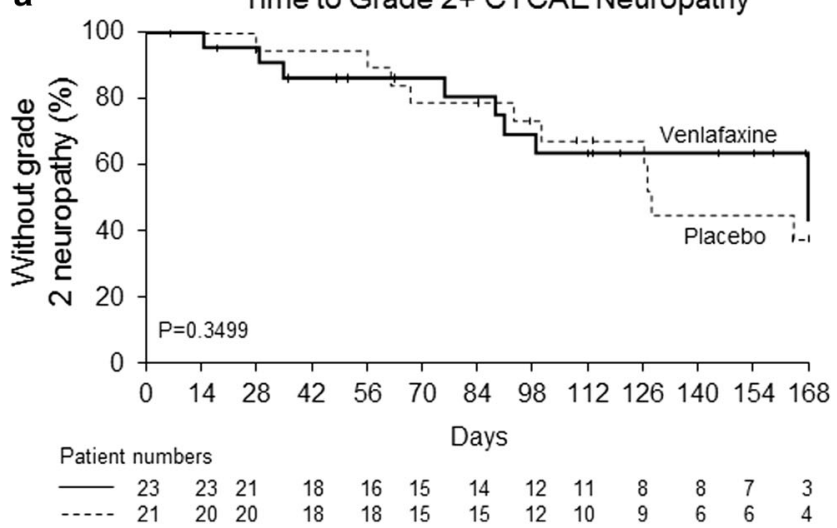

b Time to Grade 2+ Oxaliplatin-Specific Neuropathy

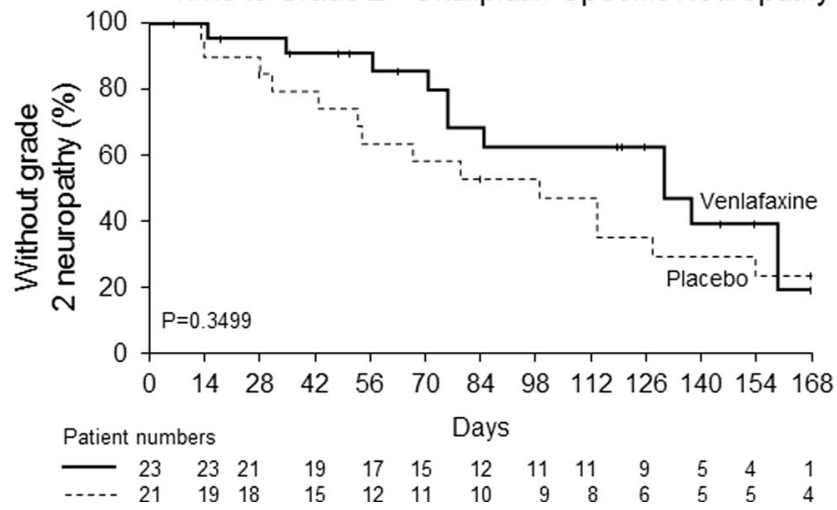

Fig. 5 Time to grade 2 or worse chemotherapy-induced peripheral neuropathy measured by the NCI-CTCAE criteria (a) and the oxaliplatin-specific neuropathy instrument (b)

over time (Fig. 6c), all numerically favoring the placebo arms. Correspondingly, the placebo group had a numerically larger mean cumulative oxaliplatin dose ( $716 \mathrm{vs} 631 \mathrm{mg}, p=0.35$ ).

\section{Evaluation of venlafaxine toxicity}

There was no suggestion of any differences, between the study arms, for nausea, anxiety, agitation, somnolence, hypertension, or allergic reactions. Grades 3 and 4 toxicities were rare in both arms. One patient in each arm experienced grade 3 hypertension. Severe neutropenia was reported in three patients in the placebo arm and five in the venlafaxine arm. There were no severe gastrointestinal toxicities noted in either group. Patient-reported symptoms were not significantly different between arms even though placebo patients had numerically worse diarrhea symptoms.

\section{Discussion}

Do the data from this study support the conduct of a larger phase III trial to investigate the use of venlafaxine for the prevention of oxaliplatin induced peripheral neuropathy?
Patients continuing to use oxaliplatin

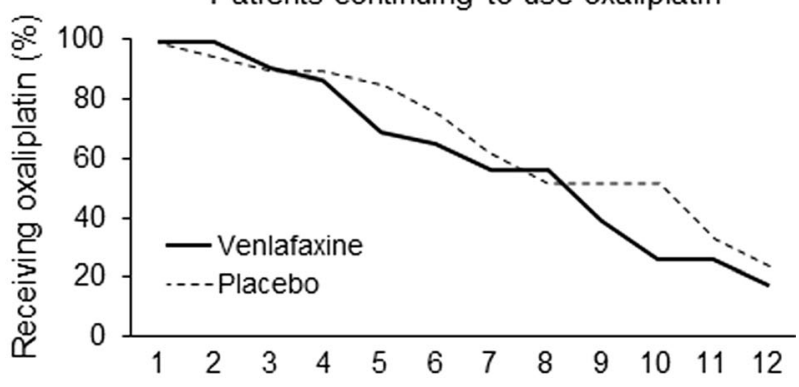

Patients receiving full oxaliplatin dose
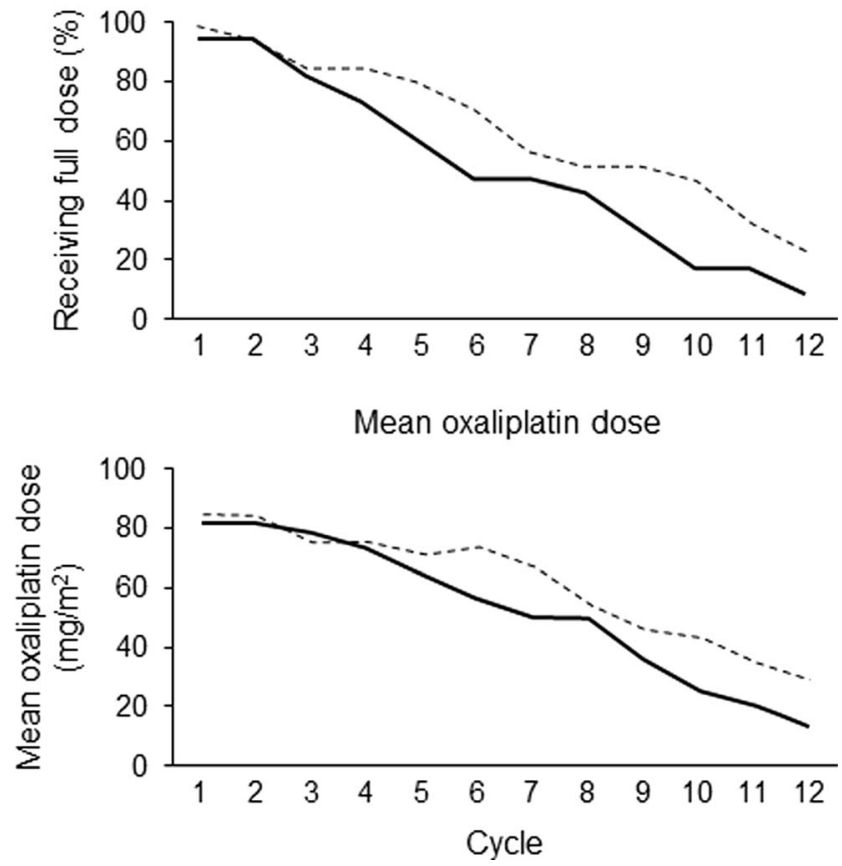

Fig. 6 Oxaliplatin use in each study arm as measured by the following: percentage of patients continuing to use oxaliplatin over time (a), percentage of patients using full doses of oxaliplatin over time (b), and mean oxaliplatin dose over time (c). In each situation, the denominators are the numbers of evaluable patients who started each study arm

There was a suggestion of some small benefit for venlafaxine, when evaluated by the Oxaliplatin Specific Neurotoxic grading scale and by acute neuropathy measures of (1) discomfort swallowing cold liquids and (2) throat discomfort, the latter observed only for the first two oxaliplatin doses. These positive trends, however, were outweighed by a lack of any such trends in the other measurements, including the following: (1) the EORTC QLQ-CIPN20 sensory, motor, or autonomic neuropathy subscales; (2) any of the individual items of the EORTC QLQ-CIPN20 tool; (3) the NCI CTCAE tool; (4) the evaluations of the doses of oxaliplatin that were able to be administered; and (5) the bulk of the acute neuropathy data. In fact, some of these other items tended to favor the placebo arm. Thus, the current study does not support the conduct of a larger phase III trial to investigate the use of venlafaxine for the prevention of oxaliplatin-induced peripheral neuropathy. 
Why do the results from the current study contrast with those reported by Durand et al. [28]? Although the current study had similarities to these previously published data, including the randomized design and size, there are some differences between these studies. First, the current trial utilized venlafaxine extended release $37.5 \mathrm{mg}$ twice daily during the duration of FOLFOX treatment, while the EFFOX trial utilized a single dose of short acting venlafaxine $50 \mathrm{mg} 1 \mathrm{~h}$ prior to oxaliplatin infusion and then venlafaxine extended release $37.5 \mathrm{mg}$ twice daily from day 2 to day 11 . Thus, the venlafaxine dose in the current trial was actually higher than that used in the former trial, as it did not have drug-free days. Dose differences between the two trials do not rationally explain the different results.

Second, the inclusion criteria for the study by Durand et al. required patient reporting of distressing neurotoxicity after administration of an initial dose of oxaliplatin. At study initiation, subjects had already received a median of 4 cycles of oxaliplatin in the venlafaxine arm and 5 cycles in the placebo arm. The current study did not allow any prior neurotoxic chemotherapy nor pre-existing neuropathy, and all patients initiated treatment prior to cycle 2 of FOLFOX. Perhaps, these inclusion criteria differences might have resulted in more patients at-risk for neuropathy in the former trial, as data demonstrate patients with more severe acute neuropathy develop more chronic neuropathy [4, 29-31]. However, it is not clear that inclusion criteria differences explain the different study outcomes.

Third, the Durand et al. trial utilized the 12 question patient-completed Neuropathic Pain Symptom Inventory. This tool is known to evaluate spontaneous ongoing pain, spontaneous paroxysmal pain, paraesthesias/dysesthesias, and the temporal sequence of pain [32]. Functional impairment was queried separately. This tool was not specifically developed to measure CIPN, understanding that, at the time the Durand et al. trial was conducted, less was known about using patient-specific neuropathy tools. The EORTC QLQCIPN20 tool evaluates sensory, motor, and autonomic symptoms with a particular focus on functional impairment and has been validated in patients receiving chemotherapy $[25,26]$. The current study used both patient- and clinician-reported measures of neurotoxicity, with relatively close agreements between the two methodologies. It is possible that the current trial was better able to measure CIPN.

Was the negative result of the current trial due to the use of a venlafaxine dose that was too low? Randomized trial data support improved efficacy for venlafaxine in the setting of painful neuropathy at higher doses than were utilized in the current study [33]. A commonly recommended venlafaxine dose, when used for painful neuropathy, is $75 \mathrm{mg}$ of extended release twice daily [34]. Venlafaxine has a high affinity for the 5-HT transporter but a relatively lower affinity for norepinephrine transporter, compared to other SNRIs. At lower doses, it is felt to act as a selective serotonin reuptake inhibitor (SSRI) [35] and may not have the same activity against painful neuropathy. The current study's lower venlafaxine dose was chosen based on the Durand et al. data and the desire to not add additional toxicity to the current study patients receiving chemotherapy. At higher doses, venlafaxine toxicity including nausea and hypertension is more pronounced and may compound chemotherapy-related side effects during treatment.

In total, the data from the current trial do not support conducting a phase III study of venlafaxine in this population. Further work is needed to identify other agents with potential efficacy for the prevention of CIPN.

Acknowledgments Research reported in this publication was supported in part by the National Cancer Institute of the National Institutes of Health under Award Number CA037447 to the legacy Cancer and Leukemia Group B (CALGB, now the Alliance for Clinical Trials in Oncology). The content is solely the responsibility of the authors and does not necessarily represent the official views of the National Institutes of Health. ClinicalTrials.gov Identifier: NCT01611155

Conflict of interest The authors have declared no conflict of interest.

\section{References}

1. Andre T, Boni C, Mounedji-Boudiaf L et al (2004) Oxaliplatin, fluorouracil, and leucovorin as adjuvant treatment for colon cancer. N Engl J Med 350:2343-2351

2. de Gramont A, Figer A, Seymour M et al (2000) Leucovorin and fluorouracil with or without Oxaliplatin as first-line treatment in advanced colorectal cancer. J Clin Oncol Off J Am Soc Clin Oncol 18:2938-2947

3. Raymond E, Chaney SG, Taamma A, Cvitkovic E (1998) Oxaliplatin: a review of preclinical and clinical studies. Ann Oncol Off J Eur Soc Med Oncol / ESMO 9:1053-1071

4. Pachman DR, Qin R, Seisler DK et al (2014) Clinical course of patients with Oxaliplatin-associated neuropathy: N08CB (alliance). ASCO Meet Abstr 32:3595

5. McKeage MJ (1995) Comparative adverse effect profiles of platinum drugs. Drug Saf 13:228-244

6. Screnci D, McKeage MJ (1999) Platinum neurotoxicity: clinical profiles, experimental models and neuroprotective approaches. J Inorg Biochem 77:105-110

7. Adelsberger H, Quasthoff S, Grosskreutz J et al (2000) The chemotherapeutic Oxaliplatin alters voltage-gated $\mathrm{Na}(+)$ channel kinetics on rat sensory neurons. Eur J Pharmacol 406:25-32

8. Gamelin L, Capitain O, Morel A et al (2007) Predictive factors of Oxaliplatin neurotoxicity: the involvement of the oxalate outcome pathway. Clin Cancer Res Off J Am Assoc Cancer Res 13:63596368

9. Weickhardt A, Wells K, Messersmith W (2011) Oxaliplatininduced neuropathy in colorectal cancer. J Oncol 2011:201593

10. Kannarkat G, Lasher EE, Schiff D (2007) Neurologic complications of chemotherapy agents. Curr Opin Neurol 20:719-725

11. Malik B, Stillman M (2008) Chemotherapy-induced peripheral neuropathy. Curr Neurol Neurosci Rep 8:56-65

12. Melli G, Taiana M, Camozzi F et al (2008) Alpha-lipoic acid prevents mitochondrial damage and neurotoxicity in experimental chemotherapy neuropathy. Exp Neurol 214:276-284 
13. Hershman DL, Lacchetti C, Dworkin RH et al (2014) Prevention and management of chemotherapy-induced peripheral neuropathy in survivors of adult cancers: american society of clinical oncology clinical practice guideline. J Clin Oncol Off J Am Soc Clin Oncol 32:1941-1967

14. Hochster HS, Grothey A, Childs BH (2007) Use of calcium and magnesium salts to reduce Oxaliplatin-related neurotoxicity. J Clin Oncol 25:4028-4029

15. Grolleau F, Gamelin L, Boisdron-Celle M et al (2001) A possible explanation for a neurotoxic effect of the anticancer agent Oxaliplatin on neuronal voltage-gated sodium channels. J Neurophysiol 85:2293-2297

16. Gamelin L, Boisdron-Celle M, Delva R et al (2004) Prevention of Oxaliplatin-related neurotoxicity by calcium and magnesium infusions: a retrospective study of 161 patients receiving Oxaliplatin combined with 5-fluorouracil and leucovorin for advanced colorectal cancer. Clin Cancer Res Off J Am Assoc Cancer Res 10:40554061

17. Grothey A, Nikcevich DA, Sloan JA et al (2011) Intravenous calcium and magnesium for Oxaliplatin-induced sensory neurotoxicity in adjuvant colon cancer: NCCTG N04C7. J Clin Oncol Off J Am Soc Clin Oncol 29:421-427

18. Loprinzi CL, Qin R, Dakhil SR et al (2014) Phase III randomized, placebo-controlled, double-blind study of intravenous calcium and magnesium to prevent Oxaliplatin-induced sensory neurotoxicity (N08CB/alliance). J Clin Oncol Off J Am Soc Clin Oncol 32: 997-1005

19. Saarto T, Wiffen PJ (2007) Antidepressants for neuropathic pain. Cochrane Database Syst Rev 17, CD005454

20. Durand JP, Alexandre J, Guillevin L, Goldwasser F (2005) Clinical activity of venlafaxine and topiramate against Oxaliplatin-induced disabling permanent neuropathy. Anti-Cancer Drugs 16:587-591

21. Durand JP, Brezault C, Goldwasser F (2003) Protection against Oxaliplatin acute neurosensory toxicity by venlafaxine. AntiCancer Drugs 14:423-425

22. Ozdogan M, Samur M, Bozcuk HS et al (2004) Venlafaxine for treatment of chemotherapy-induced neuropathic pain. Turk J Cancer 34:110-113

23. Durand JP, Deplanque G, Montheil V et al (2012) Efficacy of venlafaxine for the prevention and relief of Oxaliplatin-induced acute neurotoxicity: results of EFFOX, a randomized, double-blind, placebo-controlled phase III trial. Ann Oncol Off J Eur Soc Med Oncol / ESMO 23:200-205
24. Pocock SJ, Simon R (1975) Sequential treatment assignment with balancing for prognostic factors in the controlled clinical trial. Biometrics 31:103-115

25. Postma TJ, Aaronson NK, Heimans JJ et al (2005) The development of an EORTC quality of life questionnaire to assess chemotherapy-induced peripheral neuropathy: the QLQ-CIPN20. Eur J Cancer 41:1135-1139

26. Lavoie Smith EM, Barton DL, Qin R et al (2013) Assessing patientreported peripheral neuropathy: the reliability and validity of the European organization for research and treatment of cancer QLQCIPN20 questionnaire. Qual Life Res Int J Qual Life Asp Treat Care Rehab 22:2787-2799

27. Levi FA, Zidani R, Vannetzel JM et al (1994) Chronomodulated versus fixed-infusion-rate delivery of ambulatory chemotherapy with Oxaliplatin, fluorouracil, and folinic acid (leucovorin) in patients with colorectal cancer metastases: a randomized multiinstitutional trial. J Natl Cancer Inst 86:1608-1617

28. Durand JP, Deplanque G, Gorent J et al (2009) Efficacy of venlafaxine for the prevention and relief of acute neurotoxicity of Oxaliplatin: results of EFFOX, a randomized, double-blinded, placebo-controlled prospective study. J Clin Oncol 27:9533

29. Argyriou AA, Cavaletti G, Briani C et al (2013) Clinical pattern and associations of Oxaliplatin acute neurotoxicity: a prospective study in 170 patients with colorectal cancer. Cancer 119:438-444

30. Velasco R, Bruna J, Briani C et al (2014) Early predictors of Oxaliplatin-induced cumulative neuropathy in colorectal cancer patients. J Neurol Neurosurg Psychiatry 85:392-398

31. Park SB, Goldstein D, Lin CS et al (2009) Acute abnormalities of sensory nerve function associated with Oxaliplatin-induced neurotoxicity. J Clin Oncol Off J Am Soc Clin Oncol 27:1243-1249

32. Bouhassira D, Attal N, Fermanian J et al (2004) Development and validation of the neuropathic pain symptom inventory. Pain 108 : $248-257$

33. Rowbotham MC, Goli V, Kunz NR, Lei D (2004) Venlafaxine extended release in the treatment of painful diabetic neuropathy: a double-blind, placebo-controlled study. Pain 110:697-706

34. Dworkin RH, O'Connor AB, Audette J et al (2010) Recommendations for the pharmacological management of neuropathic pain: an overview and literature update. Mayo Clin Proc 85: S3-14

35. Stahl SM, Grady MM, Moret C, Briley M (2005) SNRIs: their pharmacology, clinical efficacy, and tolerability in comparison with other classes of antidepressants. CNS spectrums 10:732-747 